\title{
Entre l'Adour et la Baïse (partie occidentale du département du Gers, France) : une importante source de matières premières siliceuses du Sénonien
}

Marie-Roger Séronie-Vivien, Micheline Séronie-Vivien, Pascal Foucher, Dominique Millet et Françoise Millet

\section{(2) OpenEdition}

Journals

Édition électronique

URL : http://journals.openedition.org/paleo/2444

DOI : $10.4000 /$ paleo.2444

ISSN : 2101-0420

Éditeur

SAMRA

Édition imprimée

Date de publication : 15 décembre 2012

Pagination : 357-366

ISSN : $1145-3370$

Référence électronique

Marie-Roger Séronie-Vivien, Micheline Séronie-Vivien, Pascal Foucher, Dominique Millet et Françoise Millet, « Entre l'Adour et la Baïse (partie occidentale du département du Gers, France) : une importante source de matières premières siliceuses du Sénonien », PALEO [En ligne], 23 | 2012, mis en ligne le 29 avril 2013, consulté le 28 juillet 2020. URL : http://journals.openedition.org/paleo/2444 ; DOI : https:// doi.org/10.4000/paleo.2444

Ce document a été généré automatiquement le 28 juillet 2020

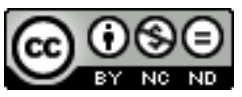

PALEO est mis à disposition selon les termes de la licence Creative Commons Attribution - Pas d'Utilisation Commerciale - Pas de Modification 4.0 International. 


\section{Entre l'Adour et la Baïse (partie occidentale du département du Gers, France) : une importante source de matières premières siliceuses du Sénonien}

Marie-Roger Séronie-Vivien, Micheline Séronie-Vivien, Pascal Foucher, Dominique Millet et Françoise Millet

La région étudiée couvre la partie occidentale du département du Gers et déborde légèrement sur les contrées voisines, Landes et Lot-et-Garonne (fig. 1). Elle s'identifie, $\mathrm{du}$ point de vue géographique, à la portion la plus occidentale du plateau du Lannemezan. Ce plateau correspond à un immense cône détritique, alluvial, résultant du démantèlement des Pyrénées pendant le Plio-Pleistocène. Cet édifice sédimentaire culmine à $660 \mathrm{~m}$ mais il s'abaisse rapidement dans le département du Gers passant de $440 \mathrm{~m}$ au sud à moins de $180 / 150 \mathrm{~m}$ au nord (Joanne 1893). L'érosion postdépositionnelle de ces sédiments alluvionnaires a épargné des lambeaux résiduels sur les parties sommitales des reliefs actuels. Ce sont ces milieux naturels qui font aujourd'hui l'objet d'exploitations agricoles, (vignobles, champs de tournesol, de maïs ou autre céréale) (fig. 2) dans lesquelles il est possible de collecter de nombreux galets dégagés du substratum (fig. 3). Parfois, d'anciennes exploitations locales de graviers rendent possible l'observation stratigraphique. 
Figure 1 - Localisation de la zone prospectée.

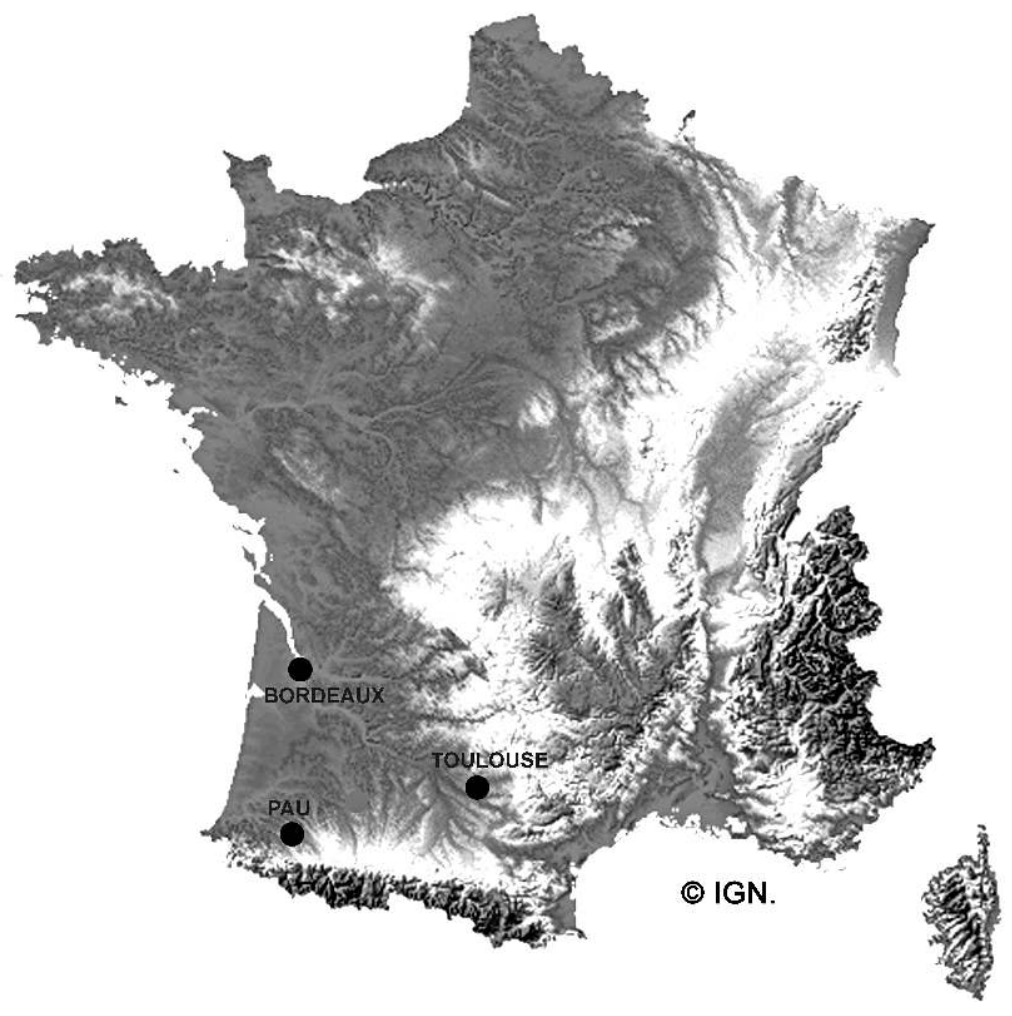

Figure 2 - Exemple de champ labouré contenant des galets de silex.

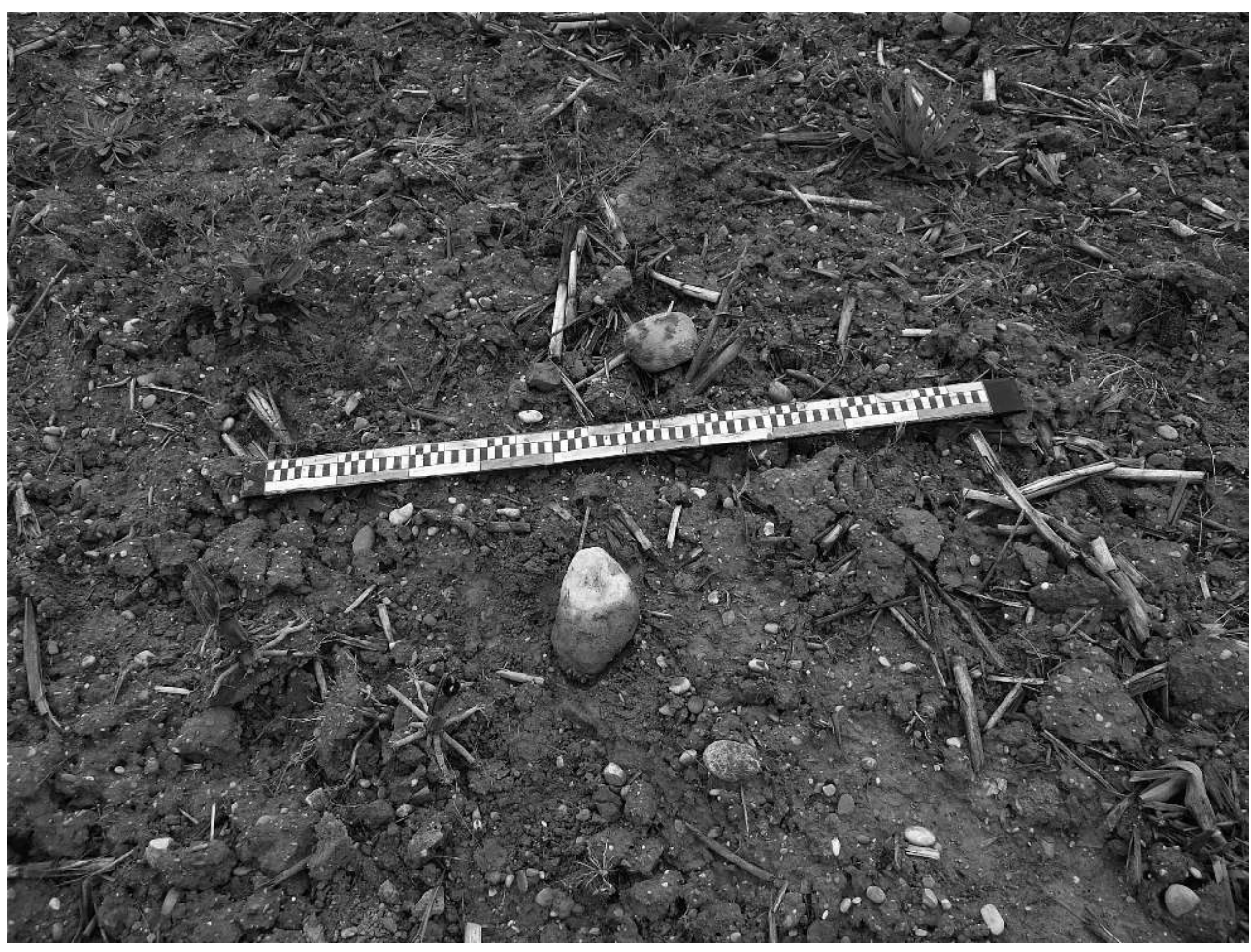


Figure 3 - Front d'exploitation d'une carrière contenant de nombreux galets de silex.

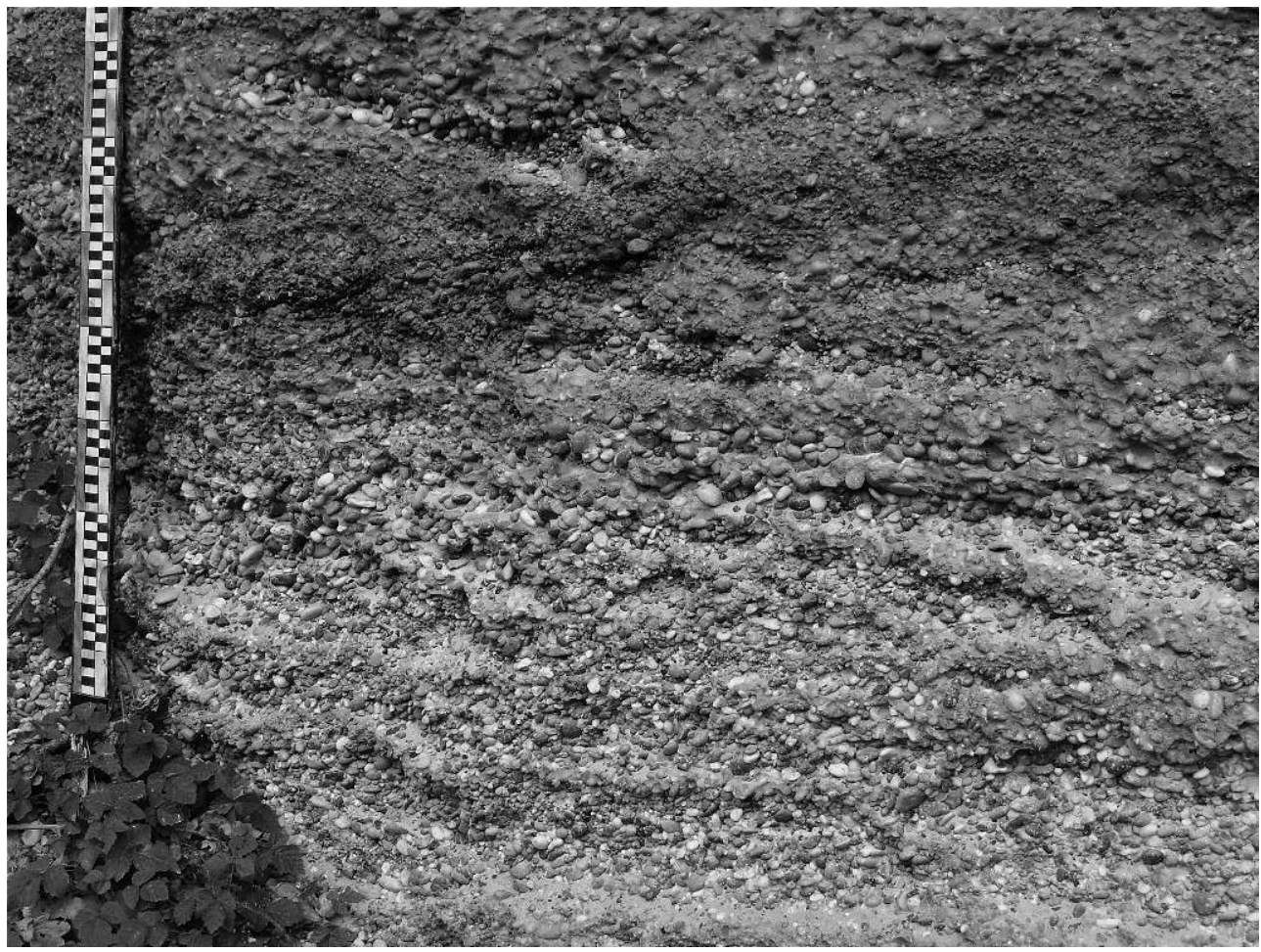

2 Des programmes de prospections systématiques de surface concernant les occupations anciennes (Paléolithique inférieur et moyen) dans la partie occidentale du Gers (Armagnac) entre 1996 et 2006 (Millet et al. 2006) nous ont permis d'identifier la présence de matières premières locales à partir d'affleurements d'extension limitée (grès quartzitiques de type Cazalège, silicifications de la Ténarèze) et d'éléments exogènes (silex du Sénonien dont certains à lépidorbitoïdes). Dès lors, après la découverte de plusieurs épandages de silex inédits, nous avons orienté notre travail de terrain vers des problématiques gîtologiques (Millet et al. 1999, 2000, 2003, 2004, 2005) selon une approche systémique.

3 Il devenait nécessaire de clarifier l'origine de ces sédiments clastiques en silex sénoniens, entre autres. Plusieurs hypothèses étaient envisageables :

- introduction à partir de gîtes périphériques à la région étudiée ;

- affleurements locaux sur des anticlinaux à cœur du Crétacé supérieur ;

- présence en position dérivée dans les alluvions quaternaires ;

- existence de concentrations ponctuelles, localisées, dans les dépôts pliocènes locaux (glaises bigarrées/argiles à galets) formant des glacis détritiques de plus en plus résiduels du sud au nord de la région étudiée.

4 L'absence de Sénonien affleurant ou sub-affleurant élimine la seconde hypothèse (dépouillement d'une centaine de minutes de sondages entre Vic-en-Bigorre et Eauze). La distribution des secteurs échantillonnés positivement dans la vallée de l'Adour (commune de Riscle) indique des potentialités au sein des alluvions rapportés au Pleistocène moyen (rognons réguliers à néocortex fluviatile). Vers le nord, hors du couloir alluvial, les rognons sont fréquemment rencontrés dans les altérites qui colmatent les têtes de vallons au pied des formations des argiles à galets et dans les paléochenaux qui incisent le sommet de sables fauves entre Gélise et Midou (fig. 4). Il 
devenait donc évident qu'il existait au moins deux origines locales excluant toute intrusion systématique de nature anthropique à partir des régions périphériques.

Figure 4 - Localisation des lieux de prélèvements (cercles).

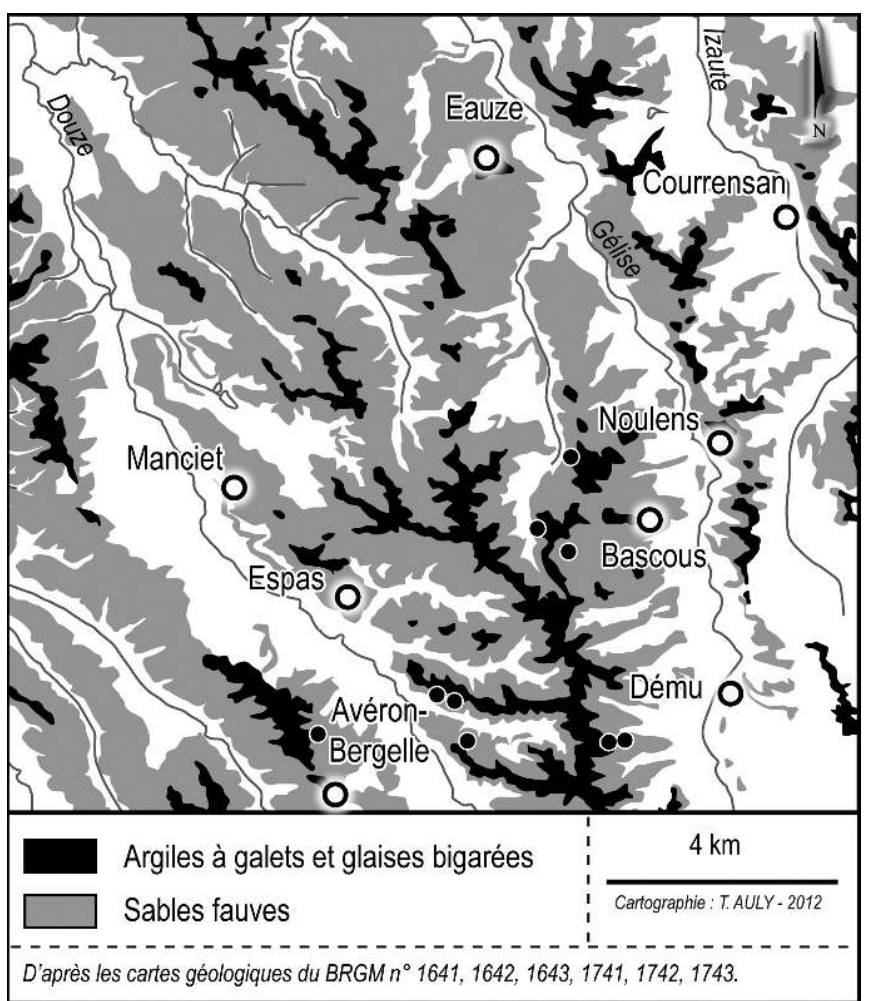

5 Le bilan géomorphologique dynamique destiné à élaborer un scénario concernant la mise en place des ces « placers » de silex sénoniens privilégie une diffusion par transits successifs de ces matériaux sous régime fluviatile de moyenne à faible compétence (dépôts en chenaux tressés et anastomosés) lors de la mise en place des formations des argiles à galets/glaises bigarrées (Pliocène). Ces dépôts, résidualisés par l'érosion, constituent des lobes distaux des glacis d'accumulation détritiques d'avant piémont centrés sur le sud-ouest du Gers. Bien que les gisements primaires ne soient actuellement pas identifiés, ces derniers ne peuvent être localisés qu'en amont des vastes cônes alluviaux de Ger et du Lannemezan.

6 A partir de ces observations géomorphologiques, des prospections de surface ont été réalisées. Elles nous ont permis de collecter 290 galets qui ont fait l'objet d'une étude pétrographique détaillée selon notre méthode habituelle (Séronie-Vivien M. et M.R. 1987 ; Séronie-Vivien M.R. 2010). Parallèlement, il a été possible d'analyser 132 artefacts (Paléolithique supérieur à Néolithique) qui proviennent de la même région et qui nous ont été aimablement prêtés par leurs propriétaires. L'inventaire pétrographique des galets a conduit à décrire et caractériser 19 types lithologiques de silex. L'attribution stratigraphique de ces types a pu être proposée avec plus ou moins de précision selon l'abondance de la microfaune contenue dans ces roches. Les résultats obtenus sont mis en relation avec les données géologiques, de surface et de subsurface, disponibles afin d'arriver à pouvoir émettre des hypothèses sur l'origine de ces silex.

7 Il faut toutefois rappeler que, pour le préhistorien, cette source de matière première est rarement prise en compte. Il est essentiellement intéressé par la géographie des 
affleurements de matières premières tels qu'ils ont pu exister à l'époque de la collecte par l'Homme préhistorique. Le contexte géologique reste déterminant pour la compréhension de la mise en place des épandages alluvionnaires plio-pleistocènes que nous avons étudiés et qui s’étendent sur une grande partie du département du Gers.

\section{1 - Caractérisation des galets en silex}

\section{1 - La vallée de l'Adour}

8 La région prospectée dans la vallée de l'Adour se situe en aval jusqu'à Aire-sur-Adour et s'étend en amont sur une grande partie du canton de Riscle (Riscle, Saint-Mont, Labarthète, Aurensan, Viella, Lelin-Lapujolle). Au total 75 galets ont été prélevés et analysés. Ils provenaient de récoltes de surface dans des terres cultivées (champs de maïs, vignoble, etc.).

Sur la rive gauche de l'Adour (50 échantillons), trois types lithologiques de silex ont été individualisés :

- Type A: Silex homogène, à texture mudstone, ciment microcristallin, avec de nombreuses zones de recristallisation (calcédoine), quelques éléments fibreux (végétaux). Bien qu'il n’y ait pas été trouvé de fossile caractéristique, il est très probable que ce type de silex soit à rattacher au Cénozoïque.

- Type Ba : Silex à structure homogène, à texture wackestone, avec intraclastes anguleux et une microfaune assez abondante: Lepidorbitoides socialis, Lepidorbitoides $s p$, Orbitoïdés, Meandropsina vidal (fig. 5c), Siderolites vidali (fig. 5e), Fascispira sp. (fig. 5g), spicules, assez rares dasycladacées. Le cortège faunique est typique du Maastrichtien supérieur du Piémont pyrénéen.

- Type Bb : Silex de structure homogène, à texture mudstone, avec de nombreux pellets, microfaune assez abondante : Lepidorbitoides sp., Clypeorbis mamillata (fig. 5a) Siderolites vidali, assez rares Pithonella (fig. 5h), spicules, rares dasycladacées. A part le silex paraissant être d'âge tertiaire (type A) tous les autres galets seraient d'âge maastrichtien supérieur. Les différences entre les types $\mathrm{Ba}$ et $\mathrm{Bb}$ sont minimes, les biotopes semblent être les mêmes et les variantes observées ne témoignent que d'une différence du niveau d'énergie.

Sur la rive droite de l'Adour, la prospection n'a porté que sur deux sites (commune de LelinLapujolle). Il a été possible de distinguer cinq types différents à partir de 25 galets collectés en surface :

- Type $\mathrm{C}$ : Silex à structure homogène, à texture mudstone avec de rares pellets et quelques intraclastes arrondis $(300 / 500 \mu)$. La microfaune est composée de Lepidorbitoides sp., Siderolites assez rares, de bryozoaires et de spicules, auquels s'ajoutent quelques dasycladales.

- Type D : Silex à structure homogène, à texture mudstone avec de nombreux à très nombreux pellets et quelques intraclastes arrondis. La microfaune est peu diversifiée (Lepidorbitoides et spicules).

- Type E : Silex à structure homogène, à texture mudstone avec de rares pellets et une matrice microcristalline. La microfaune est restreinte à des spicules et de très rares bryozoaires. La microflore est composée de tiges de dasycladales. Il est à noter l'absence de lepidorbitoïdes.

- Type F : Silex à structure homogène et texture mudstone avec quelques pellets. La microfaune ne contient pas de lepidorbitoïdes mais seulement des spicules et de rares 
bryozoaires. Quelques fragments de dasycladacées soulignent le caractère infralittoral du milieu de dépôt.

- Type G : Silex à structure microlitée avec un granoclassement apparent et une texture mudstone à pellets et très nombreux spicules souvent orientés. Les types $C$ et $D$, de même que le type $\mathrm{Bb}$ de la série précédente, sont indiscutablement attribuables à des formations d'âge maastrichtien supérieur. Ils proviennent d'une aire de sédimentation infralittorale soumise à une relativement faible agitation des eaux.

Les types $\mathrm{E}$ et $\mathrm{F}$ dans lesquels il n'a pas été trouvé de lépidorbitoïdes et dont la microfaune ne présentent aucun élément permettant une datation précise, pourraient être à rapprocher des formations sénoniennes (Coniacien à Campanien) du piémont pyrénéen.

11 Enfin, le type G, tant par sa structure que par le granoclassement du sédiment, présente toutes les caractéristiques d'un flysch pyrénéen (Barragué et al. 2001).

à noter que sur un total de 75 galets étudiés provenant des alluvions anciennes de l'Adour, 42 étaient attribuables au Maastrichtien supérieur (56\%), 31 au Sénonien (41\%), un au Flysch crétacé et un au Cénozoïque.

\section{2 - Les vallées tributaires de l'Adour : I'Izaute, le Midour, le Midouzon, la Douze}

Au nord de la vallée de l'Adour, on observe tout un éventail de cours d'eau qui drainent le flanc nord-ouest du cône alluvial du Lannemezan. Ces rivières, d'abord orientées vers le nord, s'infléchissent ensuite vers l'ouest pour devenir tributaire de l'Adour, souvent par l'intermédiaire de la Midouze. C'est dans cette partie du département du Gers qu'ont été prospectées les terres cultivées à la recherche de galets de silex. Les communes de Bétous, Cravencères, Averon-Bergelle, Aignan, Séailles, Espas, Demu (pro parte) ont été explorées. Ont été également étudiées et échantillonnées d'anciennes carrières (gravières) qui furent exploitées dans les communes d'Espas et d'AveronBergelle. Au total 170 galets alluviaux ont été collectés puis analysés. L'étude pétrographique des échantillons a conduit à distinguer, outre les types déjà décrits à partir du matériel provenant de l'Adour, huit nouveaux types lithologiques dont la description suit :

- Type $\mathbf{H}$ : Silex à structure homogène, à texture grainstone avec gros intraclastes arrondis $(300 / 500 \mu)$, microfaune assez pauvre : rares spicules, quelques foraminifères rotalidés.

- Type I : Silex à structure homogène, à texture mudstone avec de rares pellets, des agrégats (lumps), microfaune peu diversifiée : spicules, assez fréquentes pithonelles.

- Type $\mathrm{J}$ : Silex à structure homogène, à texture mudstone à pellets, rares intraclastes arrondis, bioclastes assez fréquents, présence de spicules et d'algues dasycladales (Neomerys cf. cretacea, fig. $5 b)$.

- Type K : Silex à structure homogène, à texture mudstone avec de très abondants pellets et de petits intraclastes, spicules fréquents.

- Type L : Silex à structure homogène, à texture wackestone avec quelques pellets et intraclastes arrondis $(300 \mu)$, quelques lépidorbitoïdes (Lepidorbitoides socialis).

- Type $\mathbf{M}$ : Silex à structure homogène, à texture wackestone avec quelques pellets et intraclastes arrondis, microfaune assez rare (spicules).

- Type N : Silex à structure homogène, à texture packstone à gros intraclastes arrondis (500 $\mu$ ), rares spicules quelques rotalidés et valvulinidés. 
- Type 0 : Silex à structure homogène, à texture mudstone, avec de rares pellets, quelques spicules. Des zones assez étendues de recristallisation obèrent l'observation.

De ces nouveaux types un seul (Type L) est attribuable au Maastrichtien supérieur. Tous les autres, du type $\mathrm{H}$ au type $\mathrm{O}$, sont, faute de restes fossiles caractéristiques, à rattacher au Sénonien sensu lato. Deux types ( $\mathrm{H}$ et $\mathrm{N}$ ) se distinguent par des textures qui dénotent une sédimentation dans une zone de haute énergie nettement différente des milieux de faible énergie qui caractérisent les autres structures généralement mudstone ou plus rarement wackestone. Sur les 170 galets étudiés, 63 (37\%) seraient d'origine maastrichtienne, tandis que 106 seraient à attribuer à des formations sénoniennes (du Coniacien au Campanien), et un silex au Flysch pyrénéen.

Figure 5 - Exemples de microfaune et de microflore (l'échelle de $1 \mathrm{~mm}$ est indiquée par un trait noir, sauf pour le $n^{\circ} 5 \mathrm{~h}$ où elle ne représente que $0,5 \mathrm{~mm}$ ).

$5 a$ - Clypeorbis mamillata ;

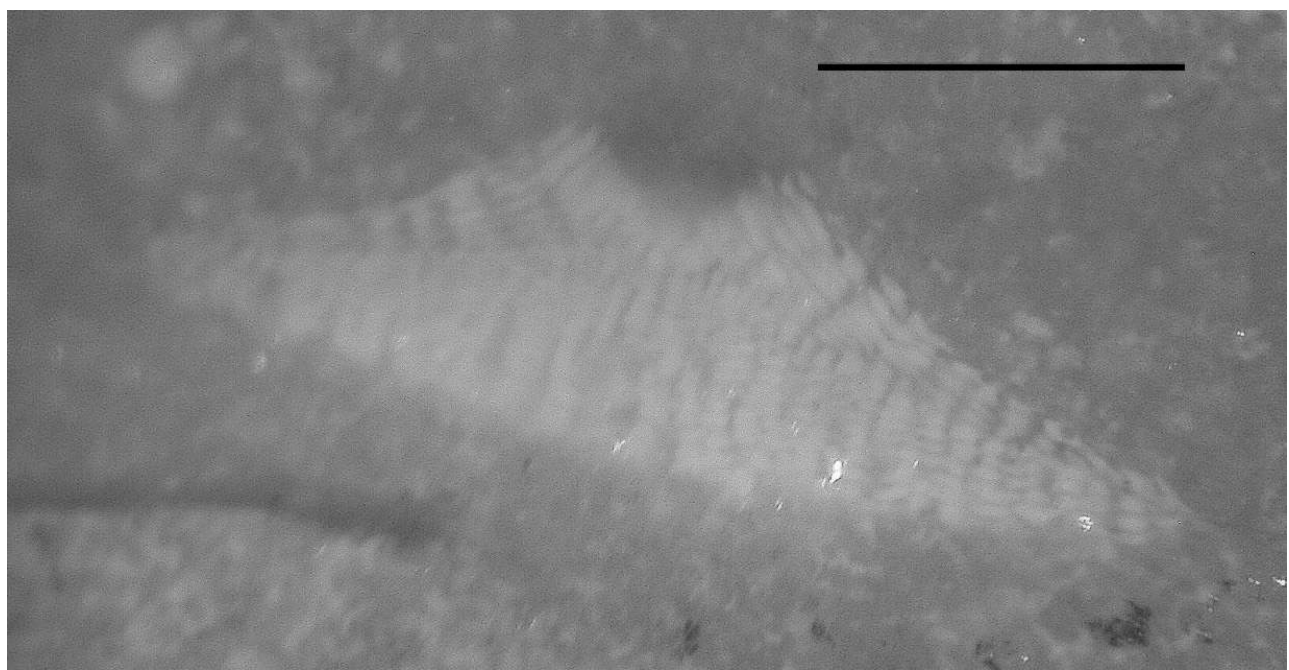


$5 b$ - Algue dasycladale - section longitudinale ;

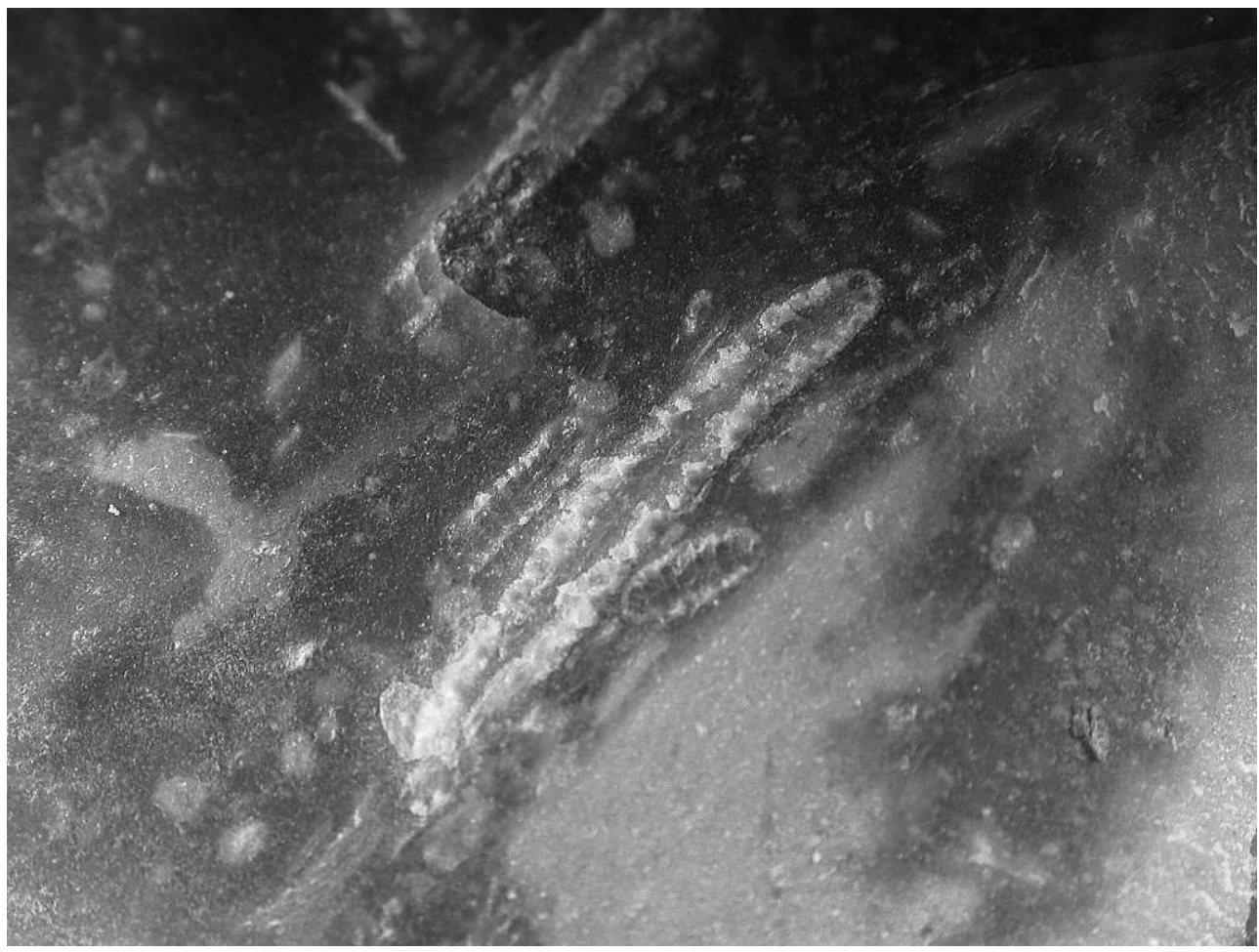

\section{5c - Meandropsina aff. vidali ;}

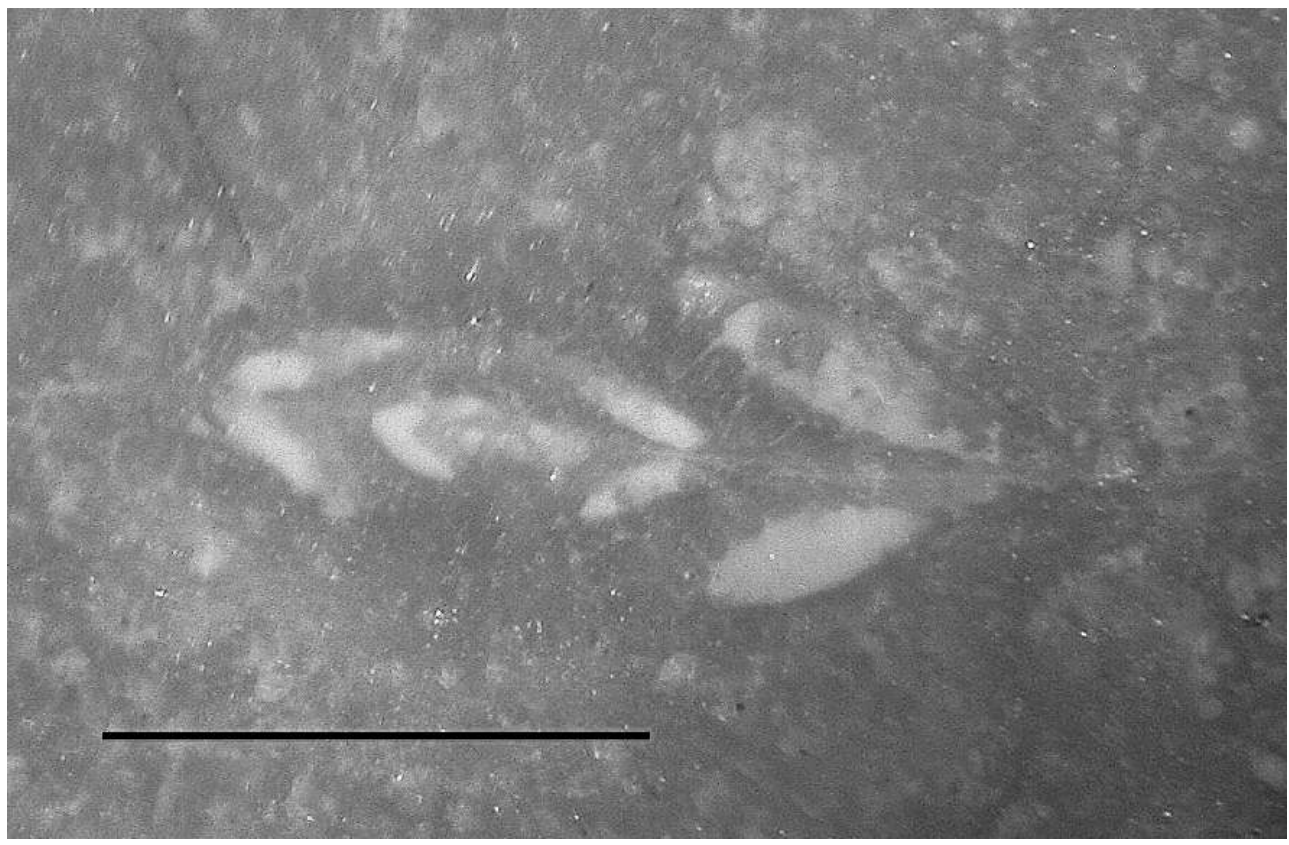


5d - Algue : dasycladacea : cf. Cylindroporella ;

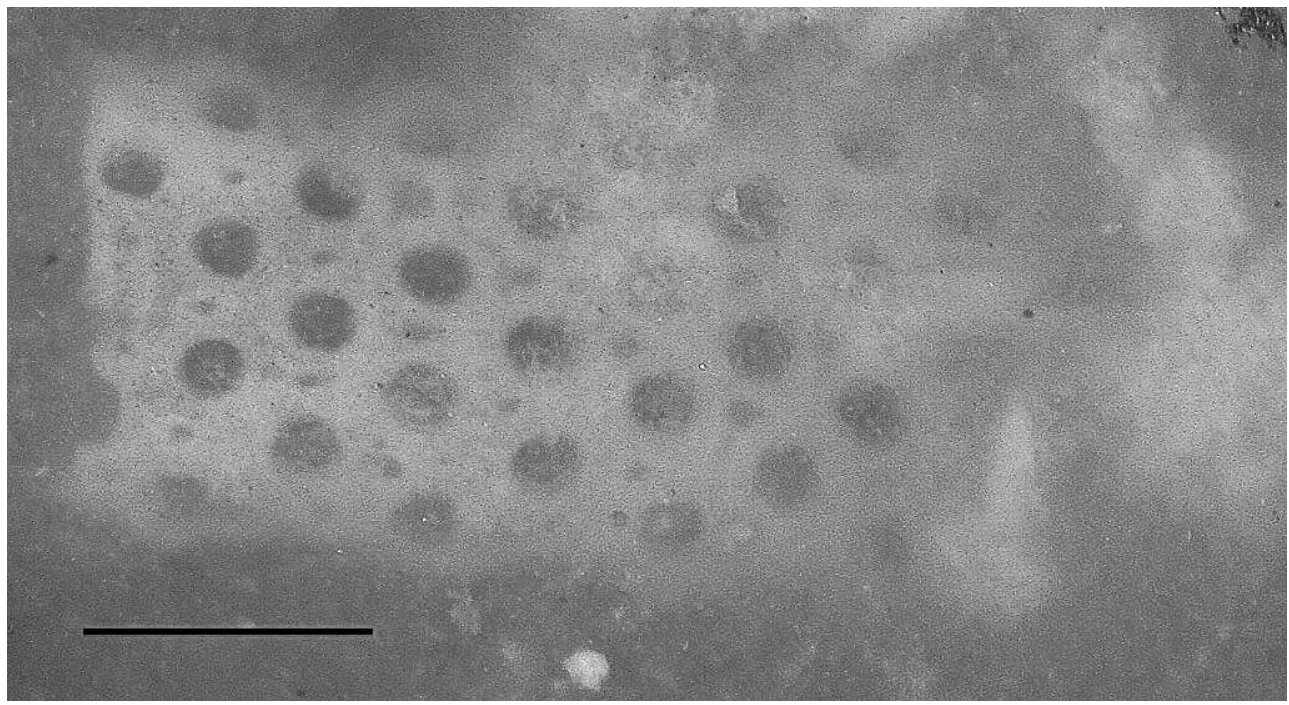

\section{$5 e$ - Siderolites vidali ;}

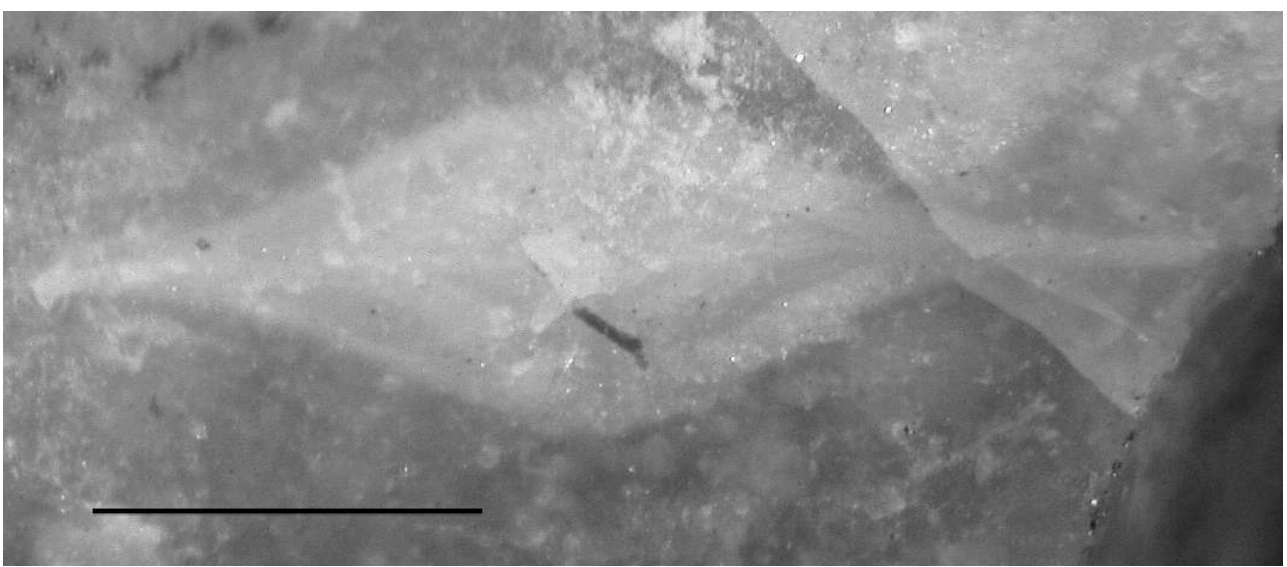


$5 f$ - Lepidorbitoides socialis ;

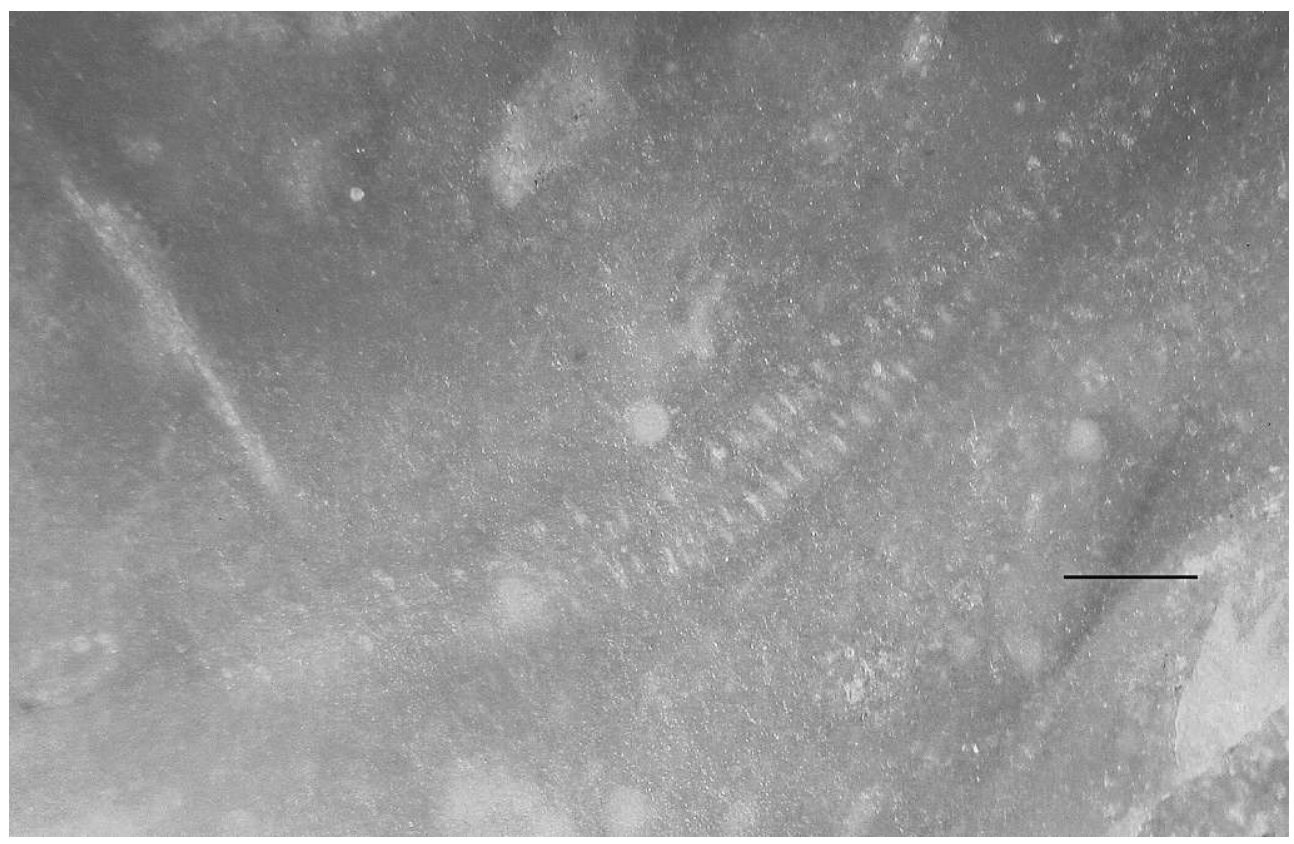

$5 g$ - Fascispira sp ;

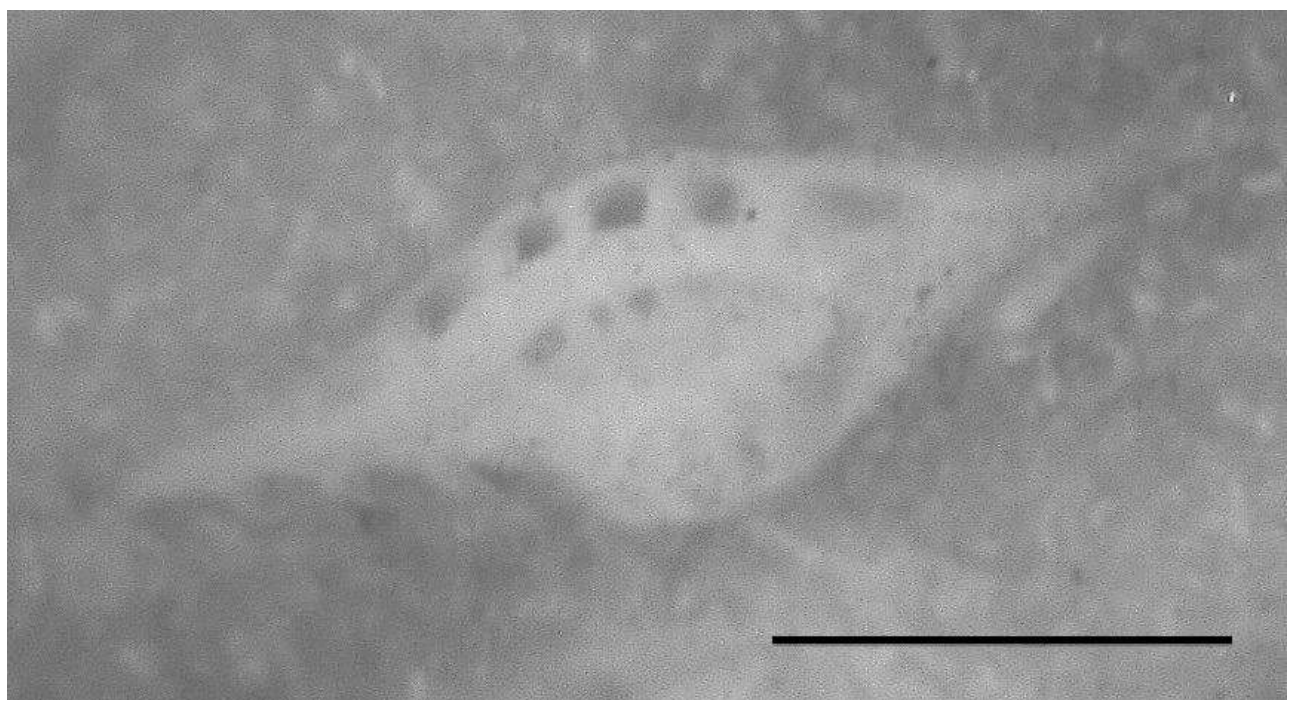


$5 \mathrm{~h}$ - cf. Pithonella.

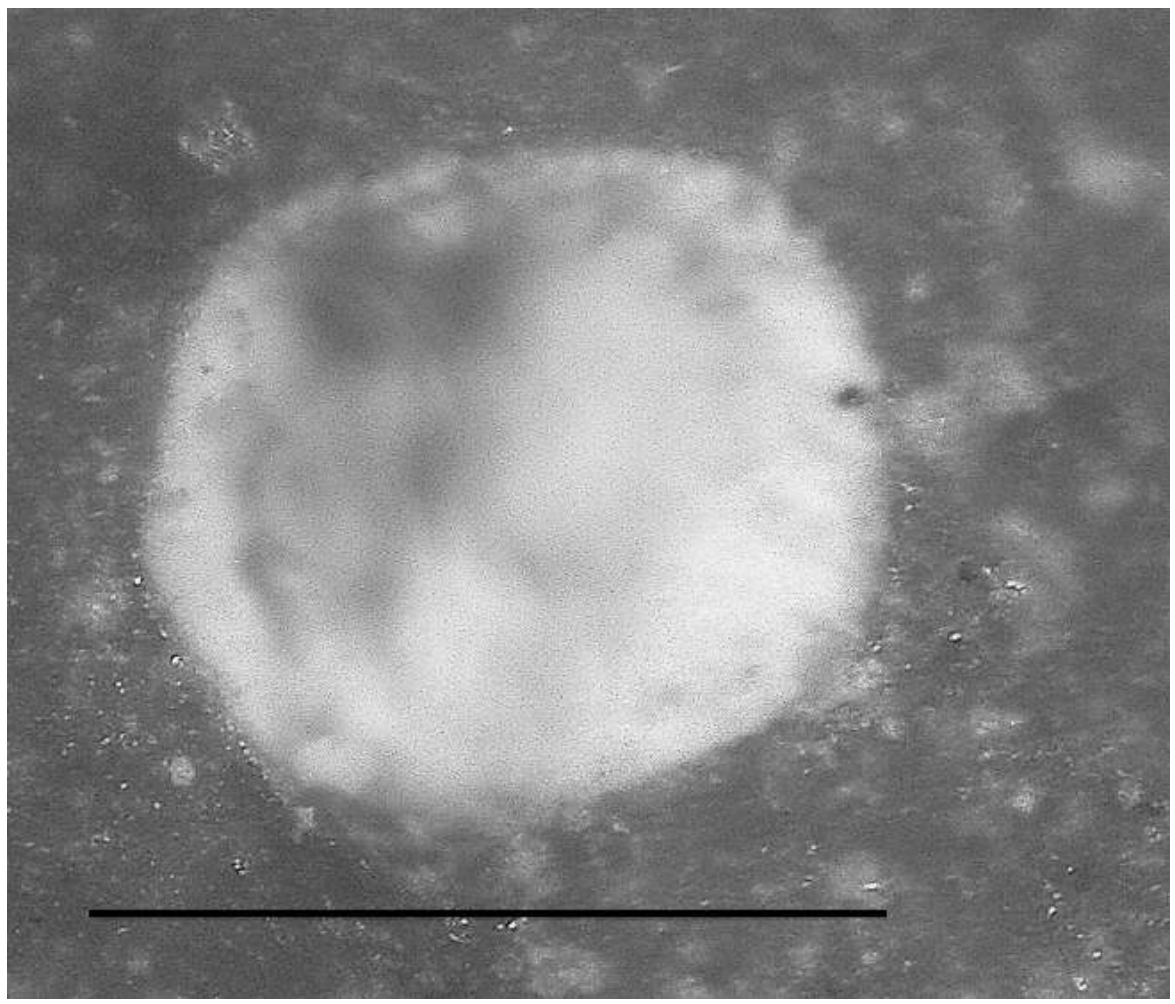

\section{3 - La vallée de la Gélise}

La Gélise est une rivière qui n'est pas tributaire de l'Adour, mais un affluent de la Garonne après sa confluence avec la Baïse. Son trajet est sensiblement sud-nord et elle ne connait pas l'inflexion vers l'ouest qui était le caractère commun de tous les cours d'eau jusqu'alors mentionnés.

La région prospectée est située dans son cours moyen sur les communes de Dému et de Bascous. C'est plus particulièrement autour de cette dernière agglomération où existent de nombreuses anciennes carrières de graves (gravières), le plus souvent abandonnées, que nous avons pu prélever un matériel abondant. Nous avons échantillonné 45 galets provenant de ces sites. Trois types nouveaux ont pu être définis :

- Type $\mathbf{P}$ : Silex à structure homogène, à texture wackestone à pellets et intraclastes anguleux, lamellibranches, peu ou pas de microfaune.

- Type $Q$ : Silex à structure microlitée, à texture grainstone à intraclastes petits $(50 \mu)$ à moyens $(300 \mu)$, calibrés et grano-classés.

- Type R : Silex à structure homogène, à texture packstone à fréquents intraclastes arrondis, assez nombreux lepidorbitoïdes (Lepidorbitoides socialis, fig. $5 f$ ).

17 Dans la population de 45 galets étudiés, on retient 25 attribuables au Sénonien, 19 (42\%) au Maastrichtien et un au Flysch pyrénéen. 


\section{2 - Étude pétrographique de silex taillés provenant de la même région}

\section{1 - La vallée de l'Adour}

Très peu d'artefacts (Paléolithique moyen, supérieur et Néolithique) ont pu être étudiés. Ils provenaient de Riscle, Saint-Mont et Lelin-Lapujolle. Ils se sont tous révélés être d'âge maastrichtien et de même facies que la plupart des galets (type Bb).

\section{2 - Les vallées tributaires de l'Adour}

Le produit des prospections anciennes menées par G. Duclos (Duclos 1991) déposé au musée d'Eauze confirme la présence d'artefacts (Paléolithique moyen à Mésolithique) exploitant les matériaux échantillonnés. Une étude pétrographique de ces collections serait à envisager.

\section{3 - La vallée de la Gélise}

- Commune de Parleboscq (Lot-et-Garonne) : La prospection de surface à la recherche de galets nous a permis de collecter 14 artefacts (Paléolithique supérieur à Néolithique). Sur ces 14 pièces, 11 sont des silex maastrichtiens (types $\mathrm{Bb}$ et $\mathrm{D}$ ), les trois autres silex sont des roches de texture mudstone à pellets contenant des spicules (Sénonien probable).

- Commune de Sainte-Maure-de-Peyrac (Lot-et-Garonne) : Un propriétaire local, Monsieur Dufaut, nous a autorisé, fort civilement, à étudier du point de vue pétrographique sa collection de silex. On y trouve des objets d'origine néolithique et paléolithique supérieur (49 pièces étudiées) et du Paléolithique moyen ou inférieur (66 silex étudiés). Les silex du Paléolithique supérieur/Néolithique comprennent 22 pièces d'âge maastrichtien (type D), les 27 autres seraient à attribuer au Sénonien (Coniacien/Campanien - types F, J, K). Parmi les 68 artefacts du Paléolithique moyen/ancien étudiés seulement trois sont en silex maastrichtiens (types $\mathrm{Ba}$ et $\mathrm{R}$ ). Tous les autres ont été confectionnés à partir de silex continentaux d'origine très locale, d'âge cénozoïque. Un contraste frappant oppose des matériaux d'étages géologiques différents : les silex du Cénozoïque continental agenais local et des galets de silex exogènes mésozoïques. Nous avons noté l'utilisation systématique d'un silex lacustre de l'Agenais (bassin de l'Auvignon) d'assez mauvais rendement dans les séries anciennes; une autre qualité provient de la vallée des abords de la Ténarèze (bassin de l'Auzoue) où affleurent des blocs volumineux de silicifications continentales conduisant à un débitage assez grossier. Par ailleurs, il existe des galets de silex plus petits, collectés dans les alluvions anciennes du bassin de la Gélise, permettant un débitage plus raffiné et l'obtention d'outils plus apprêtés, petites lamelles par exemple (Paléolithique supérieur à Néolithique). Dans ce dernier cas, bien que le nombre total d'objets étudiés soit relativement faible (115), la proportion globale de silex du Maastrichtien utilisés s'élève à $48 \%$, ce qui est du même ordre de grandeur que ce qui a été observé pour les galets des rivières du Plateau du Lannemezan que nous avons prospectés (de 37 à $56 \%$ ).

- Commune de Nérac (Lot-et-Garonne) : est actuellement en cours d'étude une collection de silex provenant d'un gisement sans doute azilien. Sur les 20 échantillons examinés, neuf sont d'âge maastrichtien, les autres étant sénoniens. 


\section{4 - La vallée de la Baïse}

gisement préhistorique du Paléolithique supérieur pour lequel il nous a été donné de faire l'étude pétrographique de 49 outils. Il s'agit du gisement de La Brette à Condom. Sur cet échantillon, 19 silex sont d'âge maastrichtien, 20 sont sénoniens et trois du Tertiaire continental.

\section{3 - Interprétation sédimentologique et paléogéographique}

Les 19 types pétrographiques décrits peuvent être distribués en un certain nombre de groupes. En fonction de caractères communs (structure, texture, composants pétrographiques, microfaune, flore, etc.). A partir de ce classement, une interprétation paléogéographique sera chaque fois proposée.

\section{1 - Cénozoïque}

\section{Groupe I (Type I)}

Silex de structure homogène, à texture mudstone, sans trace de clastes ou de fossiles si ce n'est des fragments de fibres végétales (cf. phragmites et/ou typha). Ce microfaciès est peu significatif; on peut seulement proposer une sédimentation lacustre, probablement du Cénozoïque.

\section{Groupe II (Types G et Q)}

Silex à structure microlitée avec un granoclassement des éléments détritiques. Ces critères sont spécifiques d'une sédimentation particulière que l'on rencontre dans les dépôts de type Flysch que ce soit dans les Alpes ou dans les Pyrénées. On peut donc assigner à ces silex une provenance de la bordure du front pyrénéen. On connaît des silex de ce type dans la région d'Hibarette et de Montgaillard (Barragué et al. 2001) mais il existe d'autres affleurements de silex de ce type tout au long de la fosse du Flysch pyrénéen.

\section{2 - Maastrichtien supérieur}

\section{Groupe III (Types Bb, C, D)}

Silex à structure homogène, à texture mudstone avec de fréquents à très fréquents pellets, contenant une microfaune maastrichtienne assez abondante composée d'orbitoïdés (Lepidorbitoides minor, Lepidorbitoides socialis, Clupeorbis mamillata), de sidérolites (Siderolites vidali), de lépisphères (Pithonella sp.) et de quelques algues dasycladales (Thaumatoporella, Salpingoporella, Cylindroporella) (fig. 5d), etc.

Les types $\mathrm{D}$ et $\mathrm{L}$, très proches, s'en différencient cependant par une texture wackestone qui témoigne d'un niveau d'énergie des eaux légèrement plus élevé, mais encore modeste. Ces silex paraissent s'être déposés dans un milieu marin de faible énergie, siège d'une biocénose riche en organismes généralement associés à un environnement médiolittoral peu agité. Ce faciès est typique de la sédimentation du Maastrichtien supérieur de la plateforme des Pyrénées méridionales (bassin de Lacq). 
Silex de structure homogène, à texture packstone avec de gros intraclastes arrondis et une microfaune dominée par de nombreux Lepidorbitoides socialis. Cette roche dénote un environnement nettement plus agité que le précédent avec des éléments ayant subi une action érosive et l'établissement d'un biotope pratiquement monospécifique. On peut attribuer ce sédiment à un environnement médiolittoral soumis soit à l'action de courants très actifs soit à celle des marées.

\section{3 - Sénonien (du Coniacien au Maastrichtien inférieur)}

Groupe V (Types E, F, I, J, K, O)

Silex à structure homogène, de texture mudstone avec des pellets, une matrice plus ou moins abondante, une microfaune peu diversifiée composée de spicules de spongiaires, de rares bryozoaires, de quelques pithonelles et d'algues dasycladales (Salpingoporella et Neomerys cf.cretacea). On observe des traces de remaniements (terriers, certains « lumps »). Ces observations conduisent à proposer une sédimentation dans un milieu calme de la zone infralittorale, ou même circalittorale. Aucun microfossile ne permet de situer stratigraphiquement ces niveaux, le cortège faunique conduit seulement à proposer un âge sénonien, peut-être dans sa partie supérieure (CampanienMaastrichtien) (Lacombe 1998 ).

27 Groupe VI (Types H, N )

Silex à structure homogène, de texture grainstone ou packstone, avec des intraclastes arrondis de taille atteignant $500 \mu$. La microfaune est composée exclusivement de rotalidés et de valvulinidés, ainsi que de rares spicules. Ces caractères révèlent un milieu marin à haute énergie et un milieu de dépôt sans doute circalittoral, mais la microfaune ne permet pas une attribution stratigraphique précise.

\section{4 - Discussion et conclusion}

L'étude montre que le cône détritique du Lannemezan est formé en partie d'alluvions fluviatiles qui renferment des galets de silex sénoniens (sensu lato). Ces sédiments contiennent des éléments de taille suffisante pour avoir pu servir de matière première lithique dès le Paléolithique. L'analyse détaillée du matériel alluvionnaire a montré que :

- une très faible quantité de silex d'origine lacustre est attribuable au Cénozoïque ;

- la présence, modeste, de silex qui proviendrait de formation du Flysch pyrénéen. Cette proportion très faible $(0,2 \%)$ de ce type de roche souligne la faible extension régionale des affleurements de ce genre qui ont été affectés par l'érosion du cône du Lannemezan. Cependant, il faut retenir qu'un de ces témoins a été collecté très au nord de la région étudiée, dans le cours moyen de la Gélise ;

- sur les 290 silex bruts analysés, 130 se sont avérés être du Maastrichtien supérieur (45\%) avec des teneurs relativement voisines dans les différentes vallées $(56 \%, 37 \%, 42 \%)$. Ce résultat, associé à la comparaison de la distribution géographique des types primaires, dénote un approvisionnement à partir d'une zone relativement peu étendue ;

- les inventaires effectués mettent en évidence la proportion notable du matériel lithique provenant du Crétacé supérieur. Il demeure que l'origine initiale de ces roches reste inconnue. On connaît à l'est des Pyrénées (Petites Pyrénées) et à l'Ouest (Chalosse et Ouest- 
Béarn) des structures mésozoïques qui sont encore affleurantes et qui ont été à l'origine de certaines accumulations de silex alluvionnaires. On peut supposer que des plissements prépyrénéens se poursuivent tout au long de la chaîne orogénique, en avant du front nordpyrénéen, et que le cône du Lannemezan en a érodé ou recouvert une partie entre l'Ariège et la Garonne. Des indications collectées en subsurface par les recherches pétrolières valideraient ce schéma. Par ailleurs, les affleurements de Cézan-Lavardens (Gers) et, par exemple de Créon-des-Landes, nous apprennent que des plissements pré-orogéniques sont fréquents dans la région et ne se situent pas à une grande profondeur. Comme déjà indiqué, l'origine géologique de ces matières premières lithiques ne concernent pas directement les chercheurs préhistoriens. Leur intérêt se focalise sur la distribution géographique actuelle (ou sub-actuelle) des sédiments plio-pleistocènes qui demeure leur centre d'intérêt.

En conclusion, on retiendra que les sédiments alluvionnaires du plateau du Lannemezan, produits de l'érosion fluvio-glaciaire qui a affecté les chaînons du piémont pyrénéen, contiennent un abondant matériel lithique du Crétacé supérieur. Cela a été une source de collecte au moins depuis le Paléolithique supérieur. Ce résultat serait à prendre en considération dans toute nouvelle étude de la paléogéographie des matières premières lithiques en Aquitaine. Les Petites Pyrénées et la Chalosse ne sont plus les seules sources possibles de ces silex, en particulier ceux du Maastrichtien supérieur. Des observations récentes (Lelouvier et al. 2012 ; Colonge et al. 2011) dans des sites voisins confirment cette interprétation en signalant des découvertes de silex à lépidorbitoïdes (basse vallée de la Douze) ou d'âge "vraisemblablement maestrichtien » dans la vallée du Gers, c'est-à-dire dans un cas en aval de notre zone d'étude et, dans l'autre, dans une partie plus orientale du Lannemezan dans des conditions paléotopographiques très voisines.

\section{BIBLIOGRAPHIE}

BARRAGUÉ J. et E., JARRY M., FOUCHER P., SIMONNET R. 2001 - Le silex du Flysch de Montgaillard et son exploitation sur les ateliers du Paléolithique supérieur à Hibarette (Hautes-Pyrénées), Paleo, 13, p. 29-51, 13 fig., 3 tabl.

COLONGE D., CHALARD P., BILOTTE M., DUCASSE S., PLATEL J.-P. 2011 - Nouvelle découverte d'un gîte à silex à Lepidorbitoïdesdans le Sud-Ouest de la France (Saint-Aubin, Gers) et implications archéologiques, Bulletin de la Société préhistorique française, 108, 3, p. 561-564.

DUCLOS G. 1991 - Eauze, de mémoire d'homme et de pierres, p. 21-43, In : Eauze Terre d'Histoire par Bordes M., Courtes G., Duclos G., Gugole J., Lapart J., Legrand F., Loubes G., Pedussaut P. et Vayrette A., Nogaro, Imp. Dauba, 287 p., ill.

JOANNE A. 1893 - Géographie du département du Gers. Hachette édit. 54 p., 1 carte.

LACOMBE S. 1998 - Stratégie d'approvisionnement en silex au Tardiglaciaire. L'exemple des Pyrénées centrales françaises. Bulletin Société Préhistorique Ariège-Pyrénées, 53, 223-266. 
LELOUVIER L.A., BOSC-ZANARO B., BRUXELLES L., CHALARD P., JARRY M. 2012 - En Vignes, une halte d'approvisionnement en silex au Tardiglaciaire dans le Gers (France). Bulletin de la Société Préhistorique française, 109, 1, 223-266.

MILLET D. et MILLET F. 1996 - Les formations alluviales du Pleistocène inférieur et moyen des vallées de l'Adour et de la Garonne. Premières aires d'activités humaines. Prospections thématiques (Gers et Haute-Garonne). SRA Midi-Pyrénées DFS de prospection thématiques, N.D. 55 p., 31 ill.

MILLET D., JAUBERT J., DUCLOS G., CAPDEVILLE J.-P. et al. 1999 - Une exploitation paléolithique de grès en Armagnac : le site de Cazalège à Castelnau d'Auzan (Gers). Paleo, 11, 43-70.

MILLET D. 2000 - Les matières premières siliceuses de l'interfluve Adour/Garonne : les gîtes à grès en Bas-Armagnac (Gers). In : Coord.1999 : F. Briois : Lithothèque des matières premières siliceuses, région Midi-Pyrénées, Rapport année 1999, 127-147.

MILLET D., MILLET F., CAPDEVILLE J.-P., MERCADAL P. 2003 - Technocomplexes à galets taillés du Paléolithique inférieur en Aquitaine méridiobale : moyenne vallée de l'Adour (Vic-Bilh et BasArmagnac). DFS de prospection thématique. Opération 101-103, 104 p., 28 pl.

MILLET D. et MILLET F. 2004 - Refuges naturels, matières premières siliceuses : implications paléolithiques en Gabardan et Albret méridional. SRA Aquitaine,Bilan scientifique.

MILLET D., MILLET F., DUFAUT H. 2005 - Modalités de l'anthropisation paléolithique du Bassin moyen de la Gélise en Gabardan (Landes) et Albret méridional (Lot-et-Garonne). Archéologie des Pyrénées occidentales et des Landes, t. 24, 73-84.

MILLET D. et MILLET F. 2006 - Technocomplexes à galets taillés du Paléolithique inférieur en BasArmagnac et Vic-Bilh (Gers). In : Préhistoire du Bassin de l'Adour, Colloque de Saint-Etienne de Baïgorry (12 janvier 2002)- Dir. Cl. Chauchat et al. - Comité IZPEGI, 9-32.

SÉRONIE-VIVIEN M. et SÉRONIE-VIVIEN M.R. 1987 - Les silex du Mésozoïque nord-aquitain. Approche géologique de l'étude des silex pour servir à la recherche préhistorique. Suppl.t. XV Bulletin de la Société Linnéenne de Bordeaux,135 p.

SÉRONIE-VIVIEN M.R., SÉRONIE-VIVIEN M., AFFOLTER J., DELVIGNE V., PIBOULE M., RAYNAL J.P., MONCEL M.H., TUFFERY C., TURQ A., MORALA A., ORTEGA D., X., REY M., MANGADO X., THIRY M., FOUCHER P., MILLET D. MILLET F., DUCLOS G., SCHMIDT P., FRÖHLICH F., DELAGE C., NORMAND

C. 2010 - Une Approche vers la détermination des silex : la pétrographie sédimentaire.

P. Fernandes (dir.) : Rapport d'activité du PCR « Réseau des lithothèques en Rhone-Alpes », SRA RhôneAlpes, 1 vol. 233 p.

\section{RÉSUMÉS}

La région étudiée correspond à la partie occidentale du département du Gers. Elle couvre une partie de la vallée de l'Adour, de ses affluents (Izaute, Midour, Douze) et de la vallée de la Gélise. Une étude géologique et une collecte de 290 galets alluviaux provenant des formations plioquaternaires et couvrant les parties sommitales de cette région ont été réalisées de 2010 à 2012. Parallèlement, l'examen de 132 artefacts de gisements gersois attribuables au Paléolithique supérieur a été mené. Les matériaux recueillis ont été soumis à des analyses pétrographiques, sédimentologiques et paléontologiques. Elles ont conduit à discerner 19 types lithologiques qui ont été caractérisés :

- un seul type est attribuable au Cénozoïque ;

- deux types sont caractéristiques des formations du Flysch pyrénéen ; 
- dix types paraissent attribuables au Sénonien (du Coniacien au Campanien) ;

- six types contiennent une microfaune du Maastrichtien supérieur à Lepidorbitoides.

Ces types ont été regroupés en six groupes qui mettent en valeur les niveaux stratigraphiques et les environnements paléogéographiques des différentes époques. Il en est ressorti que seulement $0,2 \%$ des galets alluvionnaires proviennent des formations crétacées du Flysch pyrénéen, $45 \%$ sont issus du Maastrichtien supérieur à Lepidorbitoides, et $54 \%$ du Sénonien (Coniacien à Campanien). Les artefacts en silex collectés dans la même région donnent des résultats très comparables (49\% de Maastrichtien).

En conclusion, on peut retenir, à ce jour, que cette partie du plateau du Lannemezan présente des zones alluvionnaires du Plio-Pleistocène qui montrent une forte teneur en galets de silex pour lesquels nous avons établi une origine géologique sénonienne et maastrichtienne. L'utilisation de ces matériaux lithiques au Paléolithique supérieur est évidente.

La mise en évidence d'une nouvelle source de matières premières dans la partie méridionale du bassin d'Aquitaine sera à prendre en compte et remet sans doute en question nombre d'interprétations antérieures.

This study refers the western part of the « Gers département » in which occurs the Adour valley and its tributaries (Izaute, Midour and Douze) and the Gelise valley. A geological study was carried out from 2010 to 2012.. It allowed us to collect 290 alluvial siliceous pebbles from the PlioPleistocene sediments. At the same time 132 flint artefacts of prehistoric sources (Upper Paleolithic) were collected. Petrographic, sedimentological and paleontological studies were carried out. They allow us to distinguish 19 lithological types :

- 1 related to the Cenozoic formation;

- 2 associated with Flysch deposits from the the Pyrenean orogenic belt ;

- 10 attributed to Senonian age ;

- 6 imputed to Maastrichtian with Lepidorbitoides.

After that these types were brought together into six sections which show their stratigraphic positions and their paleogeographical environments.

- $0,2 \%$ of the alluvial pebbles were related to the Pyrenean Flysch;

- $45 \%$ were of Uppermost Maastrichtian age (Lepidorbitoides);

- $54 \%$ were of Senonian age (Coniacian to Middle Maastrichtian).

On the other hand, the artefacts collected show a very similar proportions of siliceous pebbles ( $49 \%$ of Maastrichtian with Lepidorbitoides).The result of this study is that, up to now, the western part of the Lannemezan Plateau includes alluvial areas of Plio-Pleistocene age, containing a large amount of flint pebbles of Upper Cretaceous age. Gathering of this siliceous raw material by palaeolithic communities is obvious.It is also patent that to find out a new lithic raw material located in the southern Aquitaine will be to be retained into account, while former interpretations have to be amended.

\section{INDEX}

Keywords : Gers department, Petrography Sedimentology and Micropaleontology, Senonian, Uppermost Maastrichtian, Upper Paleolithic

Mots-clés : département du Gers, pétrographie, sédimentologie, micropaléontologie, Sénonien, Maastrichtien supérieur, Paléolithique supérieur 


\section{AUTEURS}

\section{MARIE-ROGER SÉRONIE-VIVIEN}

125 avenue d'Eysines, 33110 Le Bouscat, France - rseronie@club-internet.fr.

PASCAL FOUCHER

SRA Midi-Pyrénées, 32 rue de la Dalbade, Toulouse Cedex 6, France -

pascal.foucher@culture.gouv.fr

\section{DOMINIQUE MILLET}

UMR 5199, Université Bordeaux 1, PACEA/PPP, avenue des facultés, 33405 Talence, France prfmillet@orange.fr.

\section{FRANÇOISE MILLET}

13, rue Bordas, 33400 Talence, France - prfmillet@orange.fr 